\title{
Odontogenic differentiation of vascular endothelial growth factor-transfected human dental pulp stem cells in vitro
}

\author{
WEN ZHANG, WEI LIU, JUNQI LING, ZHENGMEI LIN, YAN GAO, XUELI MAO and YUTAO JIAN \\ Department of Operative Dentistry and Endodontics, Guanghua School and Hospital of Stomatology and Guangdong \\ Province Key Laboratory of Stomatology, Sun Yat-sen University, Guangzhou, Guangdong 510055, P.R. China
}

Received September 24, 2013; Accepted May 23, 2014

DOI: $10.3892 / \mathrm{mmr} .2014 .2481$

\begin{abstract}
Dental pulp stem cells (DPSCs) can be induced towards odontogenic differentiation. Previous studies have shown that vascular endothelial growth factor (VEGF) is able to induce the osteogenic differentiation of cells, but the effectiveness of VEGF in the odontogenic differentiation of DPSCs remains unclear. This study aimed to investigate the effects of lentivirus-mediated human VEGF gene transfection on the proliferation and odontogenic differentiation of human DPSCs in vitro. DPSCs were transfected with either lentiviral pCDH-CMV-MCS-EFI-copGFP ( $\mathrm{pCDH}$ ) vector or recombinant $\mathrm{pCDH}-\mathrm{VEGF}$ vector, and the growth characteristics of the resulting DPSCs/Vector and DPSCs/VEGF were subsequently assessed. The odontogenic differentiation genes of the two groups of cells, including alkaline phosphatase, osteocalcin, dentin sialophosphoprotein and dentin matrix protein 1 (DMP1), were evaluated by quantitative polymerase chain reaction (qPCR). The specific proteins of odontogenic differentiation, including dentin sialoprotein and DMP1, were analyzed by western blotting. DPSCs/VEGF showed similar proliferation characteristics to DPSCs/Vector during the observation period. qPCR results showed that the relative VEGF gene expression was significantly higher in DPSCs/VEGF than that in DPSCs/Vector two days after transfection $(\mathrm{P}<0.01)$. Similarly, western blot analysis showed that the protein expression levels of VEGF were higher in DPSCs/VEGF than those in DPSCs/Vector. On the first, fourth, eighth and 16th days after lentivirus-mediated transfection, the expression of odontogenic differentiation-specific genes and proteins was
\end{abstract}

Correspondence to: Professor Junqi Ling, Department of Operative Dentistry and Endodontics, Guanghua School and Hospital of Stomatology and Guangdong Province Key Laboratory of Stomatology, Sun Yat-sen University, 56 Lingyuanxi Road, Guangzhou, Guangdong 510055, P.R. China

E-mail: lingjq@mail.sysu.edu.cn

Key words: dental pulp stem cells, vascular endothelial growth factor, gene transfection, odontogenic differentiation higher in DPSCs/VEGF than that in DPSCs/Vector. These results indicated that lentivirus-mediated VEGF gene transfection promoted the odontogenic differentiation of human DPSCs in vitro.

\section{Introduction}

Dental pulp stem cells (DPSCs) are primarily isolated from dental pulp tissues $(1,2)$. These cells exhibit multilineage differentiation potential, particularly odontogenic differentiation potential. Previous studies have indicated that DPSCs are capable of differentiating into an odontoblastic lineage in vitro and forming ectopic pulp-dentine-like tissue in vivo (2-4). However, limitations remain for the direct application of DPSCs in dental tissue regeneration, and optimized issue engineering strategies are required to improve the odontogenic differentiation capacity of DPSCs (5).

The administration of cytokines is one of the most commonly utilized strategies to strengthen the osteogenic differentiation capacity of cells. Vascular endothelial growth factor (VEGF) is an important cytokine that can promote the osteogenic differentiation of cells. A previous study demonstrated that recombinant human VEGF improved the cell proliferation and alkaline phosphatase (ALP) expression of dental pulp cells (6). VEGF culture medium also promoted the osteogenic differentiation capacity of MC3T3 stem cells by increasing the expression of ALP and osteocalcin (OCN) in vitro (7). Compared with control cells, VEGF-treated human periodontal ligament stem cells exhibited a significantly higher ALP activity, which further led to the formation of increased mineralized tissue (8). Furthermore, VEGF culture medium increased ALP expression and calcium accumulation of DPSCs in vitro (9). However, there are limitations in the local delivery of cytokines, including the short half-life, large dose requirements, high costs, the necessity for repeated applications and poor expression distribution (10). Gene transfection technology may be a solution to some of these technical caveats. Transient transfection of the VEGF gene into human periosteal cells could promote the expression of ALP, OCN and Collagen I (11). VEGF gene-transfected rat bone marrow stromal cells (BMSCs) were reported to have higher ectopic osteogenesis in vivo (12). Another study also confirmed the 
improved osteogenic differentiation capacity of rat BMSCs by lentivirus-mediated VEGF gene transfection (13). Due to the similarity between osteogenic and odontogenic differentiation among DPSCs (14), lentiviral vector-mediated stable transfection of the VEGF gene may be an effective strategy to improve the odontogenic differentiation capacity of DPSCs.

This study aimed to explore the effects of lentivirus-mediated VEGF gene transfection on the odontogenic differentiation of human DPSCs in vitro. The proliferation and odontogenic differentiation capacities of VEGF gene-transfected DPSCs (DPSCs/VEGF) were analyzed. The results of this study could enhance the understanding of the biological characteristics of DPSCs/VEGF, and may provide theoretical evidence for the application of lentivirus-mediated VEGF gene transfection in dental tissue engineering.

\section{Materials and methods}

Isolation of DPSCs. Patients were recruited for this study from the Guanghua Hospital of Stomatology (Guangzhou, China). All participants provided their informed consent. The study was approved by the Ethical Review Committee of the Hospital of Stomatology, Sun Yat-Sen University (Guangzhou, China). DPSCs were isolated from freshly extracted third molars without signs of decay (Fig. 1A). The extracted teeth were thoroughly cleaned, then cut at the cementoenamel junction using a sterile dental fissure bur. The tissues in the pulp chamber were exposed and gently separated from the crown. The pulp tissues were subsequently minced and digested with $3 \mathrm{mg} / \mathrm{ml}$ collagenase type I (Worthington Biochem, Freehold, NJ, USA) and $4 \mathrm{mg} / \mathrm{ml}$ dispase (Boehringer Mannheim, Indianapolis, IN, USA) for $1 \mathrm{~h}$ at $37^{\circ} \mathrm{C}$. The cells were then passed through a $70-\mu \mathrm{m}$ strainer (BD Biosciences, Bedford, MA, USA) to generate a single-cell suspension. The isolated DPSCs were seeded at densities of between $1 \times 10^{4}$ and $1 \times 10^{5} /$ well in six-well plates (Corning, New York, NY, USA) containing Dulbecco's modified Eagle's medium (DMEM; Invitrogen Hong Kong Ltd., Hong Kong, China) supplemented with $10 \%$ fetal bovine serum (FBS; Sijiqing, Hangzhou, China), $100 \mu \mathrm{M}$ L-ascorbic acid-2-phosphate (Wako, Tokyo, Japan), 100 U/ml penicillin-G and $100 \mathrm{mg} / \mathrm{ml}$ streptomycin, and were cultured with $5 \% \mathrm{CO}_{2}$ at $37^{\circ} \mathrm{C}$.

Differentiation stimulation. The multipotent differentiation potential of the cells was identified by osteogenic and adipogenic differentiation induction (14). Briefly, the cells were exposed to osteogenic medium (DMEM supplemented with $10 \mathrm{nmol} / \mathrm{l}$ dexamethasone, $10 \mathrm{mmol} / \mathrm{l} \beta$-glycerophosphate, $50 \mu \mathrm{g} / \mathrm{ml}$ ascorbate phosphate, $10 \mathrm{nmol} / 1$ 1,25 dihydroxyvitamin D3 and $10 \% \mathrm{FBS}$ ) and adipogenic medium (DMEM supplemented with $1 \mu \mathrm{mol} / 1$ dexamethasone, $1 \mu \mathrm{g} / \mathrm{ml}$ insulin, $0.5 \mathrm{mmol} / 1$ 3 -isobutyl-1-methylxantine and 10\% FBS) for 28 days. Alizarin Red S and Oil Red O reagent were used to visualize the calcium accumulation and oil droplets, respectively.

Flow cytometric analysis. Prior to the conduction of the experiments, the phenotype of the freshly isolated DPSCs was evaluated by flow cytometry (FCM) for the expression of STRO-1/Alexa Fluor 647 (BioLegend, San Diego, CA, USA), cluster of differentiation 146 (CD146)/phycoerythrin
(PE) (BD Pharmingen, San Diego, CA, USA), CD34/PE (BD Pharmingen), CD45/fluorescein isothiocyanate (FITC; BD Pharmingen)) and CD24/FITC (BD Pharmingen).

Construction of lentivirus plasmid. The lentivirus vector pCDH-CMV-MCS-EF1-copGFP (pCDH; System Biosciences, Mountain View, CA, USA) with green fluorescent protein (GFP) label was used to visualize the recombinant plasmid expression. The human VEGF primers were designed with Oligo 7.0 software (Molecular Biology Insights, Plymouth, MN, USA) according to the National Center for Biotechnology Information GenBank no. AF486837.1, and were amplified by polymerase chain reaction (PCR). The PCR primers were designed as follows: Forward, GCCGAATTCATG AACTTTCTGCTGTCTTG (the underlined sequence indicates an EcoRI site); reverse, GCCGGATCCTCACCG CCTCGGCTTGTCAC (the underlined sequence indicates a BamHI site). For PCR amplification, specific primers were used with following reaction conditions: Pre-denaturation, $95^{\circ} \mathrm{C}$ for $3 \mathrm{~min}$; 30 cycles of denaturation, $95^{\circ} \mathrm{C}$ for $15 \mathrm{sec}$; primer annealing, $55^{\circ} \mathrm{C}$ for $30 \mathrm{sec}$; primer extension, $72^{\circ} \mathrm{C}$ for $1 \mathrm{~min}$ and final extension at $72^{\circ} \mathrm{C}$ for $7 \mathrm{~min}$, prior to storage at $4^{\circ} \mathrm{C}$ for $10 \mathrm{~min}$. The amplified products were digested using EcoRI and BamHI restriction enzymes. The fragment containing human VEGF gene was cloned into the vector to produce the pCDH-CMV-MCS-EF1-copGFP-VEGF recombinant lentiviral plasmid, referred to as pCDH-VEGF.

Gene transfection. 293FT cells (System Biosciences) were cultured in DMEM with $10 \%$ FBS, prior to being seeded $\left(1.2 \times 10^{6}\right.$ cells) into a $10 \mathrm{~cm}$ dish one day before transfection. The recombinant plasmid pCDH-VEGF, packaging plasmid psPAX.2 (Cyagen, Guangzhou, China) and envelope plasmid pMD2.G were co-transfected into the 293FT cells using Lipofectamine ${ }^{\mathrm{TM}} 2000$ (Invitrogen Life Technologies, Carlsbad, CA, USA) $(15,16)$. The liquid supernatant was collected after $48 \mathrm{~h}$, centrifuged at $1,000 \mathrm{x} \mathrm{g}$ at $37^{\circ} \mathrm{C}$ for $10 \mathrm{~min}$ and filtered using a $0.2-\mu \mathrm{m}$ syringe (Millipore, Bedford, MA, USA). The third-passage DPSCs were infected with the supernatant to acquire the recombined DPSCs/VEGF. By use of the same protocol, the blank pCDH vector was used to infect DPSCs to construct DPSCs/Vector as a negative control. Through analyzing the percentage of GFP fluorescence, the transfection ratio was identified by ImageJ software (NIH, Bethesda, MD, USA). VEGF expression from the DPSCs/Vector and DPSCs/VEGF was assessed using a quantitative PCR (qPCR) and western blot analysis two days after transfection. The DPSCs/Vector and DPSCs/VEGF were seeded in $25-\mathrm{cm}^{2}$ culture flasks containing DMEM supplemented with $10 \% \mathrm{FBS}$, and cultured with $5 \% \mathrm{CO}_{2}$ at $37^{\circ} \mathrm{C}$. The culture medium was changed at 24-h intervals.

Cell proliferation assessment. DPSCs/Vector and DPSCs/VEGF were seeded onto 96-well plates at the density of $2 \times 10^{3}$ cells/well and cultured in DMEM with $10 \%$ FBS. The cell proliferation was evaluated using a Cell Counting kit 8 (CCK8) according to the manufacturer's instructions (Dojindo Molecular Technologies, Tokyo, Japan). The CCK8 reduction/attenuation values of the wells were measured by spectrophotometer at an optical density (OD) of $520 \mathrm{~nm}$ 
Table I. Human-specific primer sequences used for quantitative polymerase chain reaction.

\begin{tabular}{ll}
\hline Gene & \multicolumn{1}{c}{ Primer sequence (5-3') } \\
\hline VEGF & Forward: CTACCTCCACCATGCCAAGT $(\mathrm{bp})$ \\
& Reverse: AGCTGCGCTGATAGACATCC \\
ALP & Forward: CTATCCTGGCTCCGTGCTC \\
& Reverse: GCTGGCAGTGGTCAGATGTT \\
OCN & Forward: CTCACACTCCTCGCCCTATT \\
& Reverse: TTGGACACAAAGGCTGCAC \\
DSPP & Forward: GCCACTTTCAGTCTTCAAAGAGA \\
& Reverse: GCCCAAATGCAAAAATATGTAA \\
DMP1 & Forward: AAAATTCTTTGTGAACTACGGAGG \\
& Reverse: GAGCACAGGATAATCCCCAA \\
GAPDH & Forward: AAGGTGAAGGTCGGAGTCAA \\
& Reverse: AATGAAGGGGTCATTGATGG \\
\hline VEGF, vascular endothelial growth factor; ALP, alkaline phosphatase; OCN, osteocalcin, DSPP, dentin sialophosphoprotein; DMP1, dentin \\
matrix acidic phosphoprotein 1; bp, base pairs.
\end{tabular}

(BioPhotometer Plus; Eppendorf, Hamburg, Germany). The tests were performed on the first, second, fourth and eighth days after seeding. The assay of each well was repeated in triplicate.

qPCR analysis. On the first, second, fourth, eighth and 16th days after transfection, the total RNA of the DPSCs/Vector and DPSCs/VEGF was isolated using TRIzol ${ }^{\circledR}$ (Invitrogen Life Technologies), and the quantity of extracted RNA was evaluated by a spectrophotometer (BioPhotometer Plus; Eppendorf). For each sample, $2 \mu \mathrm{g}$ RNA was used to synthesize the cDNA of each gene using the RevertAid ${ }^{\mathrm{TM}}$ First Strand cDNA Synthesis kit (Thermo Scientific ${ }^{\text {TM }}$ Molecular Biology, Shenzhen, China). The qPCR reaction mix used was iQSYBR Green Supermix (BioRad, Hercules, CA, USA) and the reaction was controlled by the spectrofluorimetric thermal iCycler iQ5 (Bio-Rad). VEGF gene and four odontogenic differentiation genes, ALP, OCN, dentin sialophosphoprotein (DSPP) and dentin matrix protein 1 (DMP1), were assessed. For PCR amplification of the cDNA of each gene, an initial amplification using gene-specific primers (Table I) was performed with a denaturation step $\left(95^{\circ} \mathrm{C}\right.$ for $3 \mathrm{~min})$, followed by 39 cycles of denaturation $\left(95^{\circ} \mathrm{C}\right.$ for $10 \mathrm{sec})$, primer annealing $\left(55^{\circ} \mathrm{C}\right.$ for $\left.10 \mathrm{sec}\right)$ and primer extension $\left(72^{\circ} \mathrm{C}\right.$ for $\left.30 \mathrm{sec}\right)$. The amplification efficiency of these genes was determined relative to the housekeeping gene, GAPDH. Each sample was performed in triplicate.

Western blot analysis. DPSCs/Vector and DPSCs/VEGF were seeded on six-well plates with $5 \times 10^{4} /$ well density. On the first, second, fourth, eighth and 16th days after transfection, the cells were harvested for western blot analysis. The culture media of the DPSCs/Vector and DPSCs/VEGF were removed from the six-well plates and the cells were washed with $2 \mathrm{ml}$ phosphate-buffered saline at $4^{\circ} \mathrm{C}$ for $1 \mathrm{~min}$. The total proteins of these cells were extracted with $100 \mu 1$ radio-immunoprecipitation assay buffer (BioTeke, Beijing, China) and $1 \mu$ l phenylmethanesulfonyl fluoride phosphatase inhibitor, and then collected by centrifugation at $12,000 \mathrm{rpm}$ for $5 \mathrm{~min}$. A bicinchoninic acid protein assay kit (Thermo Fisher Scientific, Inc., Waltham, MA, USA) was used to detect the total protein concentrations of the two groups of cells. Equal quantities of protein from the samples were then subjected to $12 \%$ SDS-PAGE for electrophoresis. Thereafter, the separated proteins were transferred onto polyvinylidene difluoride membranes (Millipore, Billerica, MA, USA). The membranes were blocked with $5 \%(\mathrm{w} / \mathrm{v})$ non-fat milk for $2 \mathrm{~h}$ at room temperature, and incubated respectively with 1:500 mouse anti-human VEGF antibody (Abcam, MA, USA), rabbit anti-human DMP1 antibody and goat anti-human dentin sialoprotein (DSP) antibody (Santa Cruz Biotechnology Inc., Santa Cruz, CA, USA) overnight at $4^{\circ} \mathrm{C}$. The membranes were subsequently incubated with secondary antibody at $37^{\circ} \mathrm{C}$ for $2 \mathrm{~h}$. GAPDH was used as the internal control. The resultant films were visualized by an Enhanced Chemiluminescence Western Blotting Detection system (Millipore).

Statistical analysis. The data are presented as the mean \pm standard deviation. The two-way analysis of variance (ANOVA) test was used to analyze the differences between the DPSCs/Vector and DPSCs/VEGF (SPSS, Inc., Chicago, IL, USA). $\mathrm{P}<0.05$ was considered to indicate a statistically significant difference.

\section{Results}

Morphological, multipotent differentiation and phenotypic characteristics of DPSCs. DPSCs were isolated from third molars and a cellular suspension was obtained (Fig. 1A and B). The majority of the adherent DPSCs exhibited a spindle-like appearance with extending cytoplasmic processes (Fig. 1C). Following in vitro culture for 10 days, the first passage of DPSCs reached complete confluence (Fig. 1D). Calcium accumulation (Fig. 1E) and oil droplets (Fig. 1F) were observed in DPSCs subsequent to differentiation induction for 28 days. The 

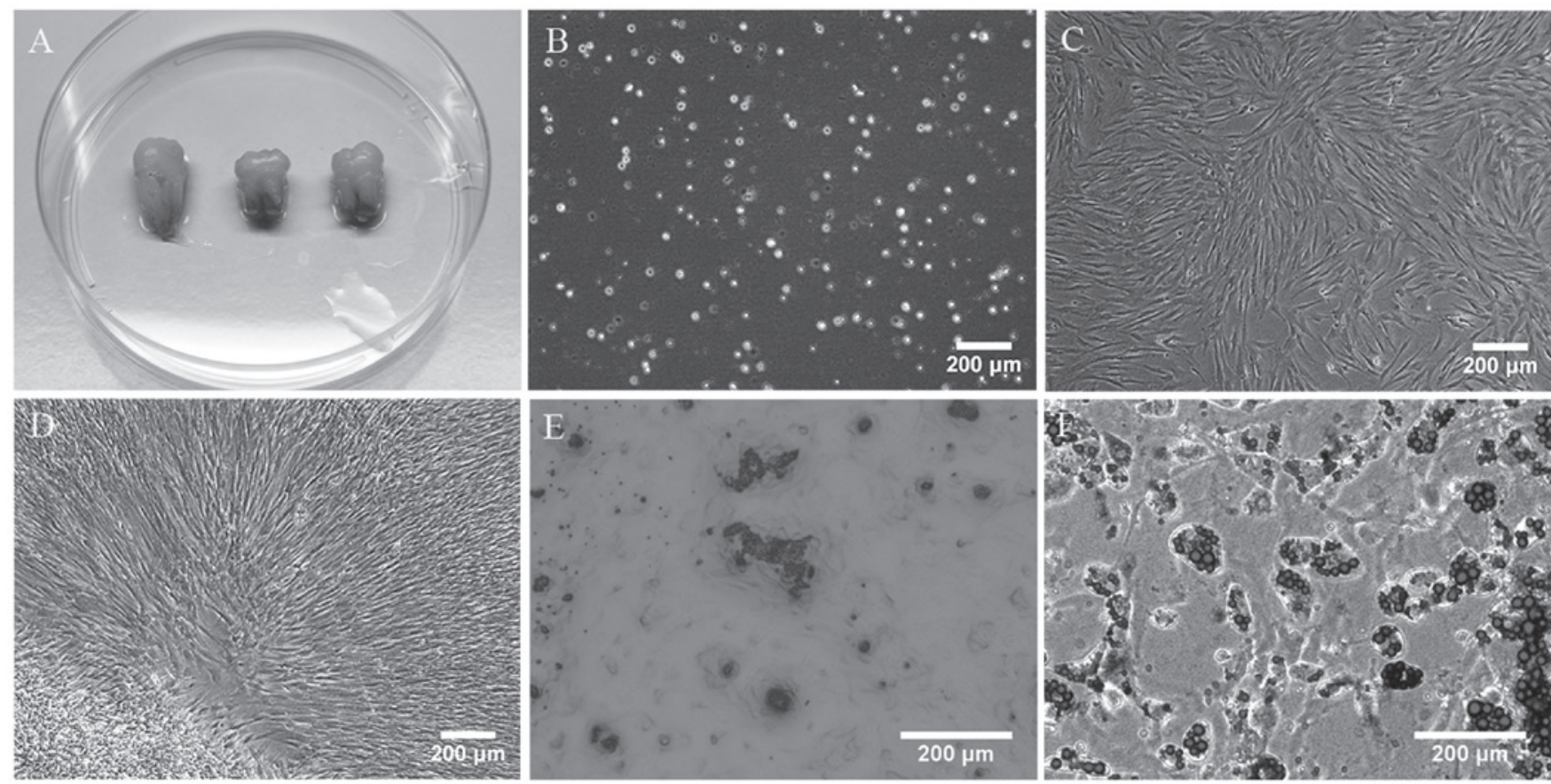

Figure 1. Isolation and identification of DPSCs. (A) DPSCs were isolated from healthy third molars. (B) Dental pulp tissue was digested, and a DPSC suspension was obtained. (C) Following three days in culture, DSPCs exhibited a spindle-like appearance with 2-4 extending cytoplasmic processes. (D) Following 10 days in culture, DSPCs reached $\sim 100 \%$ confluency. (E) Alizarin Red S staining of DPSC cultures on the 28th day of osteogenic induction. (F) Oil red O staining of DPSC cultures on the 28th day of adipogenic induction. DPSCs, dental pulp stem cells.
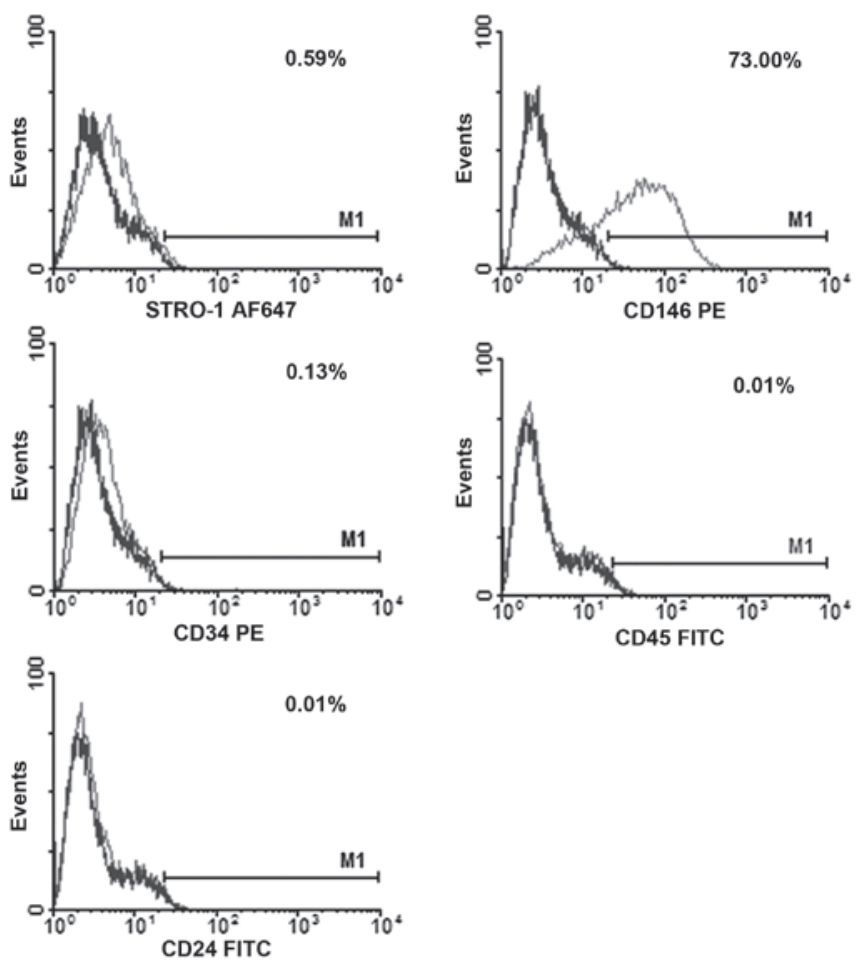

Figure 2. Flow cytometry of DPSCs. Representative flow cytometry samples for DPSC characterization are shown. The expression levels of STRO-1, CD146 and CD34 were $0.59,73.00$ and $0.13 \%$, respectively. CD, cluster of differentiation; PE, phycoerythrin; FITC, fluorescein isothiocyanate; DPSCs, dental pulp stem cells.

FCM results showed that the expression of STRO-1, CD146, CD34, CD45 and CD24 was 0.59, 73.00, 0.13,0.01 and $0.01 \%$, respectively (Fig. 2).
Transfection efficiency. The DPSCs/Vector and DPSCs/VEGF were successfully constructed (Fig. 3). The DPSCs/Vector and DPSCs/VEGF exhibited a spindle-like shape with extending cytoplasmic process, as observed in untransfected DPSCs (Fig. 3A and D). The green fluorescence was detected in the majority of the transfected cells (Fig. 3B and E), and the transfection ratio was $\sim 90 \%$ (Fig. 3C and F), analyzed using ImageI software. The VEGF gene expression was higher in the DPSCs/VEGF $(8.22 \pm 0.59)$ than that in the DPSCs/Vector $(1.00 \pm 0.06)(\mathrm{P}<0.01)$ two days after transfection $($ Fig. $3 \mathrm{G})$. The western blot analysis also showed that VEGF expression in the DPSCs/VEGF was significantly upregulated, compared with that in the DPSCs/Vector (Fig. 3H).

DPSC proliferation characteristics. A similar proliferation profile was observed for the DPSCs/Vector and DPSCs/VEGF (Fig. 4). The average OD values of the two groups of cells significantly increased from 0.2 to 0.5 during the observation period of eight days $(\mathrm{P}<0.05)$. However, larger standard deviations in the OD readings from the DPSCs/VEGF were observed when compared with those from the DPSCs/Vector over time.

Odontogenic differentiation gene expression. The relative ALP gene expression of the DPSCs/Vector and DPSCs/VEGF increased during the first eight days (Fig. 5). The relative ALP gene expression in the DPSCs/VEGF was significantly higher than that in the DPSCs/Vector on the first, fourth, eighth and 16 th days after gene transfection $(\mathrm{P}<0.05)$. The increasing trend in the relative OCN expression in the DPSCs/Vector was no longer observed on the 16th day after transfection; however, the increasing trend remained in the DPSCs/VEGF. The relative expression of OCN was statistically higher in the 

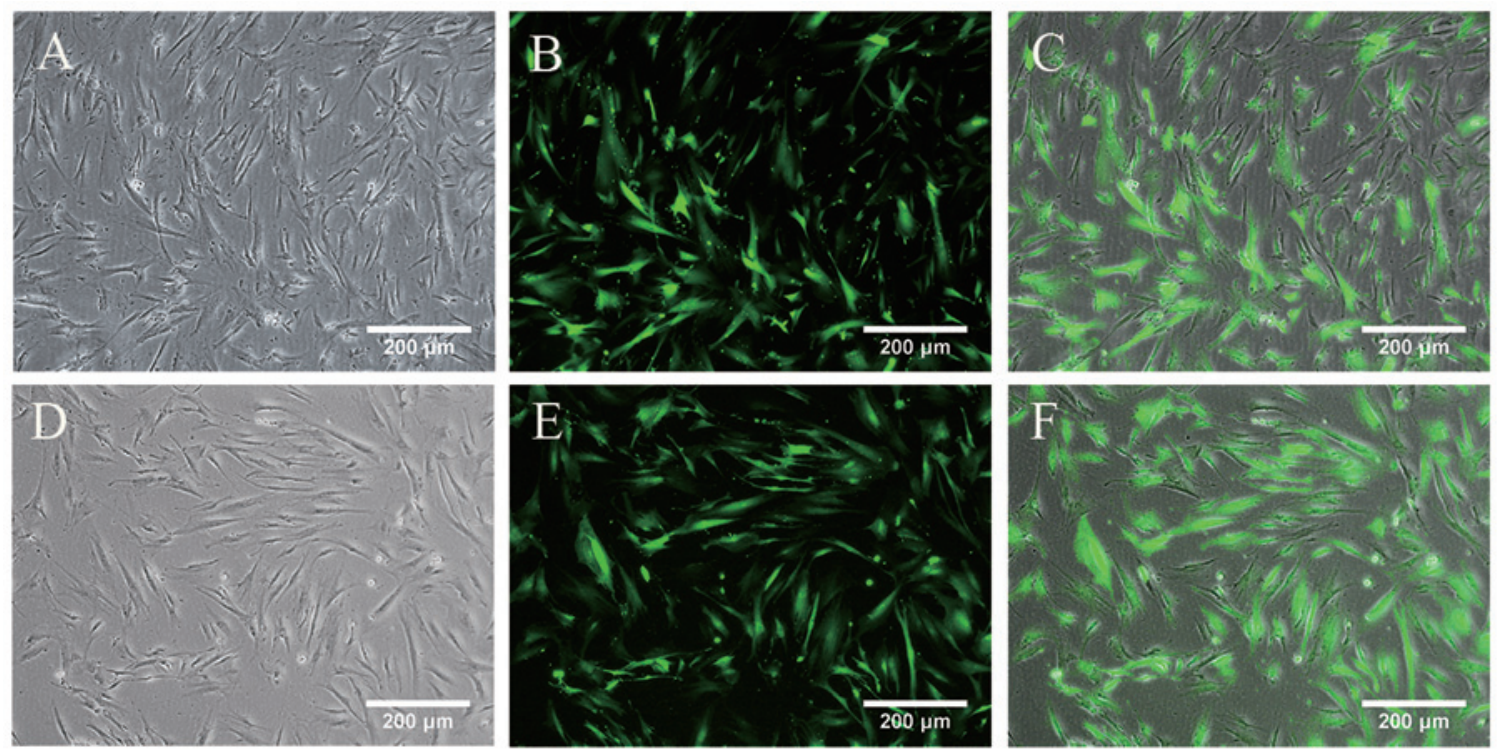

G

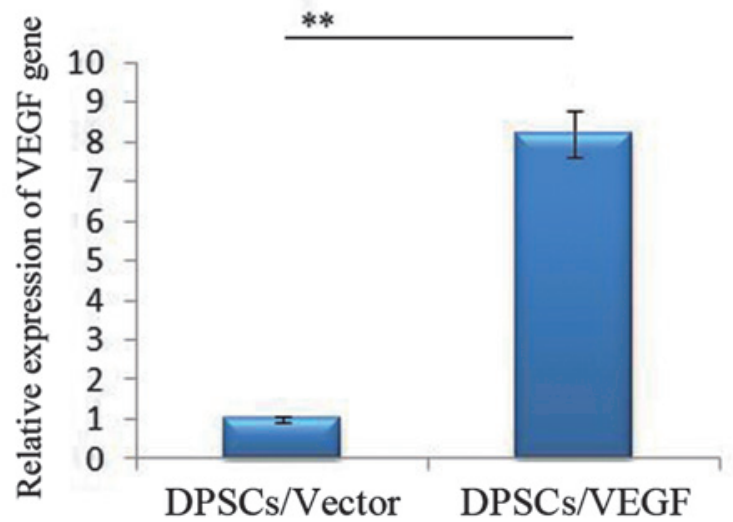

$\mathbf{H}$

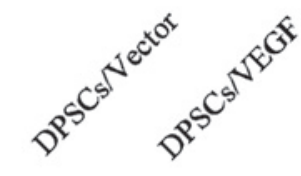

VEGF

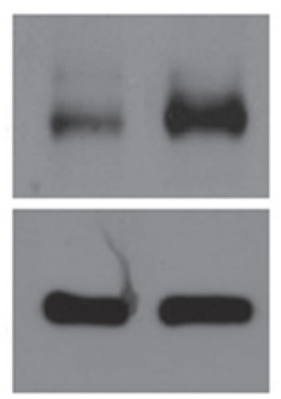

Figure 3. Lentiviral transfection efficiency. (A) DPSCs/Vector. (B) GFP fluorescence in DPSCs/Vector. (C) Merged image of (A) and (B). GFP was expressed in $89 \%$ of DPSCs/Vector. (D) DPSCs/VEGF. (E) GFP fluorescence in DPSCs/VEGF. (F) Merged image of (D) and (E). GFP was observed in 91\% of DPSCs/VEGF. (G) The relative expression of VEGF gene in DPSCs/Vector and DPSCs/VEGF was assessed by quantitative polymerase chain reaction two days after transfection. The relative expression of VEGF was statistically higher in DPSCs/VEGF than in DPSCs/Vector ("P<0.01). (H) VEGF expression in DPSCs/Vector and DPSCs/VEGF was analyzed by western blotting two days after transfection. Error bars represent the mean \pm standard deviation. VEGF, vascular endothelial growth factor; DPSCs, dental pulp stem cells; GFP, green fluorescent protein.

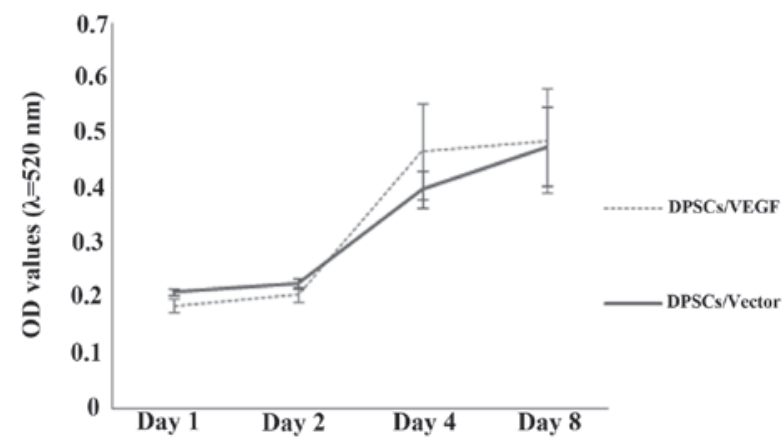

Figure 4. Cell proliferation. The proliferation of DPSCs/Vector and DPSCs/VEGF was analyzed by cell counting kit 8 on the first, second, fourth and eighth days of lentivirus-mediated gene transfection. The OD values of the two groups of cells were comparable during the eight-day observation period. Error bars represent the mean \pm standard deviation. VEGF, vascular endothelial growth factor; DPSCs, dental pulp stem cells; OD, optical density.

DPSCs/VEGF as compared with that in the DPSCs/Vector on the fourth, eighth and 16th days after gene transfection
$(\mathrm{P}<0.05)$. Both the DPSCs/Vector and the DPSCs/VEGF showed an enhancement in DSPP expression over time. The DSPP expression levels were statistically higher in the DPSCs/VEGF than those in DPSCs/Vector at the four time-points $(\mathrm{P}<0.01)$. Furthermore, the relative expression levels of DMP1 gene in the DPSCs/VEGF were significantly higher than those in the DPSCs/Vector on the first, fourth and 16th days after gene transfection $(\mathrm{P}<0.05)$. For the expression of ALP, OCN, DSPP and DMP1 genes, the two-way ANOVA showed a significant main effect for the cell types and culture times $(\mathrm{P}<0.05)$, and the interaction between the cell types and culture times was also significant $(\mathrm{P}<0.05)$.

Odontogenic differentiation protein expression. Western blot analysis confirmed that the odontogenic differentiation-specific marker DSP was significantly upregulated $(\mathrm{P}<0.05)$ in the DPSCs/VEGF as compared with the DPSCs/Vector on the first, fourth, eighth and 16th days after transfection, and the odontogenic differentiation-specific marker DMP1 was also significantly upregulated $(\mathrm{P}<0.05)$ in the DPSCs/VEGF as 

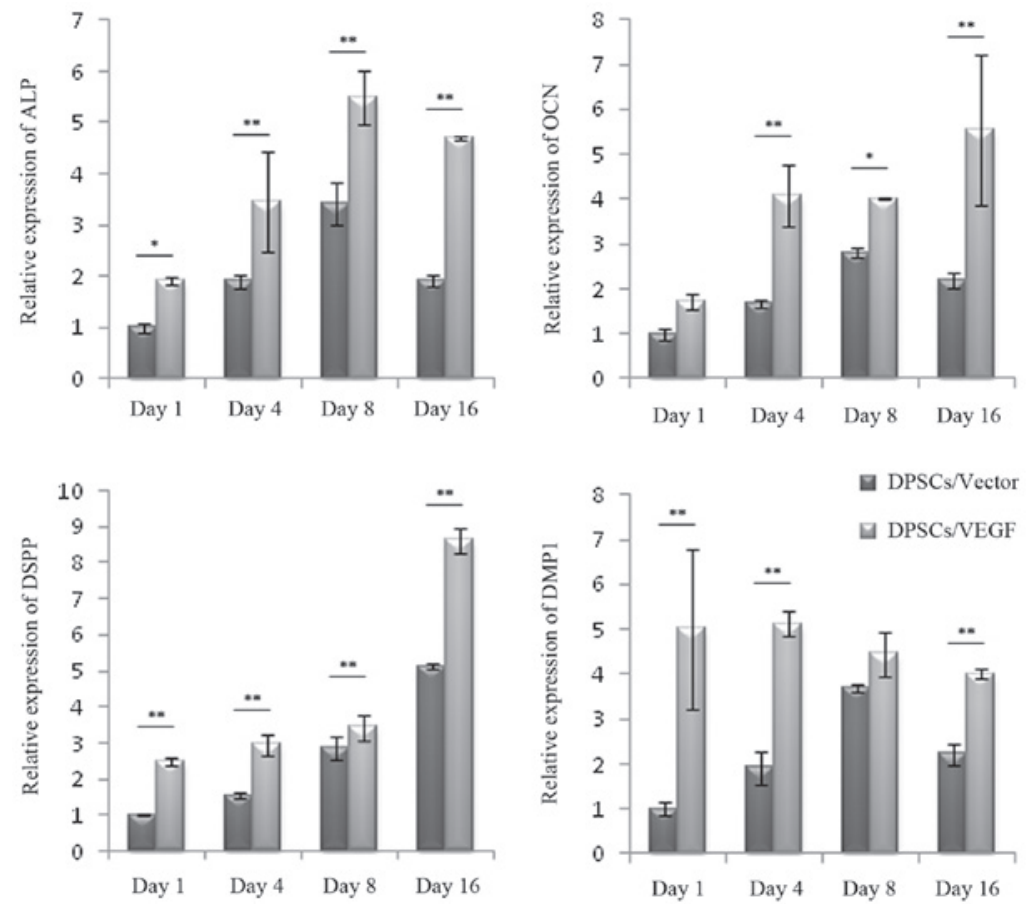

Figure 5. Odontogenic differentiation gene expression. The gene expression of DPSCs/Vector and DPSCs/VEGF was assessed by quantitative polymerase chain reaction on the first, fourth, eighth and 16th days of lentivirus-mediated gene transfection. The relative expression levels of ALP, OCN, DSPP and DMP1 genes were generally upregulated in DPSCs/VEGF as compared with those in DPSCs/Vector. ${ }^{*} \mathrm{P}<0.05$ and ${ }^{* *} \mathrm{P}<0.01$. Error bars represent the mean \pm standard deviation. VEGF, vascular endothelial growth factor; ALP, alkaline phosphatase; OCN, osteocalcin, DSPP, dentin sialophosphoprotein; DMP1, dentin matrix acidic phosphoprotein 1; DPSCs, dental pulp stem cells.

A

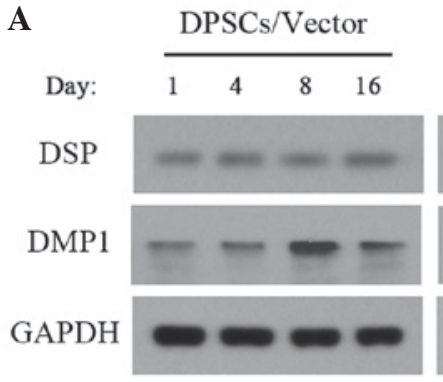

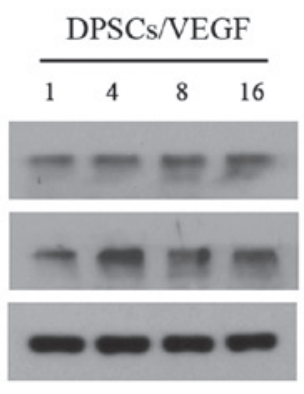
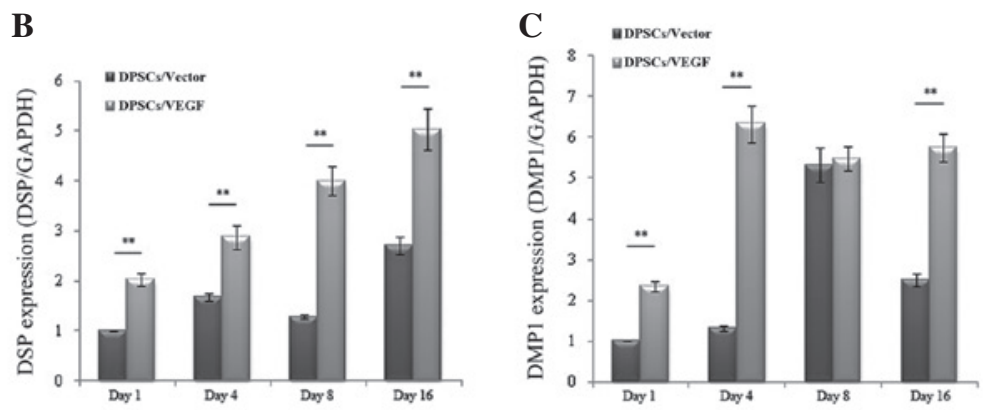

Figure 6. Odontogenic differentiation protein expression. (A) The expression levels of DSP and DMP1 in DPSCs/Vector and DPSCs/VEGF were analyzed by western blotting on the first, fourth, eighth and 16th days of gene transfection. (B) DSP and (C) DMP1 expression was significantly upregulated in DPSCs/VEGF as compared with that in DPSCs/Vector at the four time-points. ${ }^{* *} \mathrm{P}<0.01$. Error bars represent the mean \pm standard deviation. VEGF, vascular endothelial growth factor; DPSCs, dental pulp stem cells; DSP, dentin sialoprotein; DMP1, dentin matrix acidic phosphoprotein 1.

compared with the DPSCs/Vector on the first, fourth and 16th days after transfection (Fig. 6). For the expression of DSP and DMP1, two-way ANOVA showed a significant main effect for the cell types and culture times $(\mathrm{P}<0.05)$, and the interaction between the cell types and culture times was also significant $(\mathrm{P}<0.05)$.

\section{Discussion}

In the present study, evidence from the formation of calcium accumulation and oil droplets following osteogenic and adipogenic induction showed that the isolated DSPCs were multipotent. The expression of STRO-1 and CD146 in the DPSCs was 0.59 and $73.00 \%$ respectively, indicating that the isolated cells conformed to the phenotypic characteristics of
DSPCs. STRO-1 has been previously observed in pulp tissues and is regarded as a marker for stromal stem cells, recognizing the trypsin-insensitive epitope on perivascular cells (4). In the majority of previous studies, STRO-1 expression has been observed in DPSCs, with an expression level ranging between 0.46 and $26.14 \%(4,14,17)$. CD146 is a stem cell marker identified in adult bone marrow and dental pulp tissue, associated with blood vessels (18). Studies have shown positive expression of CD146 among DPSCs, with a range in expression of between 20.4 and $98.0 \%(2,14,19,20)$. CD34 is a marker expressed in the majority of stem and progenitor cells, involved in the maintenance of the plastic state of undifferentiated cells (21). The expression of CD34 in this study was $0.13 \%$, suggesting that undifferentiated cells were present in the DPSC culture. CD45 and CD24 are markers of hematopoietic/endothelial stem 
cells (14). Consistent with discussions in a previous review (4), no CD45 and CD24 expression was observed in this study.

In the present study, cell-culture conditions without L-ascorbic acid-2-phosphate and other external supplements following lentivirus-mediated transfection were selected. Cells that are cultured with L-ascorbic acid-2-phosphate have been shown to exhibit a significant improvement in cell proliferation, ALP expression and osteogenic differentiation capacity (22-25). Culturing the DPSCs with L-ascorbic acid-2-phosphate may cause hybrid bias in the analysis and confound the interpretation of the role of the VEGF expressed in the described genetically modified DPSCs.

ALP has been widely used as a marker for differentiated cells producing mineralized matrix $(7,14)$. OCN, a non-collagenous protein, is usually expressed during the late stages of osteogenic/odontogenic differentiation $(7,14)$. In this study, the expression of ALP and OCN was significantly enhanced in the DPSCs/VEGF as compared with that in the DPSCs/Vector. This result was consistent with that from other reports on cells with VEGF overexpression in vitro $(11,13)$. The expression of odontogenic differentiation-specific markers, such as DSPP and DMP1 genes, was also enhanced among the DPSCs/VEGF in this study. DSPP is a dentine non-collagenous protein, mainly expressed by odontoblasts in the mineralized nodules and organized structures (14). DMP1 plays an important role in the maturation of ameloblasts, osteoblasts and odontoblasts, as well as in the mineralization of these cells (26). The increased expression of odontogenic differentiation genes identified in this study is consistent with an improvement in the odontogenic differentiation capacity of the DPSCs due to the overexpression of VEGF.

DSP, the amino-terminal part of DSPP, is a specific protein involved in the odontogenic differentiation of DPSCs, and it is always expressed by newly-formed odontoblasts associated with the secretion of pre-dentin matrix $(27,28)$. The expression of DSP occurs prior to the initiation of the dentin mineralization, not yet present in pre-odontoblasts (27). An in vivo study has additionally shown that DSP is involved in the initiation of dentin mineralization, but not in the maturation of dentin (29). DSP appeared not only in the odontoblasts of the primary dentin but also in the odontoblast-like cells of reparative dentin. The function of DSP could be associated with the synthesis of the dentin matrix and the conversion of pre-dentin to mineralized dentin (28). In the present study, western blot analysis indicated that DSP expression in the DPSCs/VEGF was significantly higher than that in the DPSCs/Vector at the four selected time-points. Furthermore, DMP1 expression in the DPSCs/VEGF was also significantly higher than that in the DPSCs/Vector at three time-points. These results were consistent with the qPCR data, and therefore further confirmed that VEGF gene transfection improved the odontogenic differentiation of DPSCs.

In conclusion, DPSCs/VEGF were successfully constructed in the present study by lentivirus-mediated VEGF gene transfection of human DPSCs. No significant difference was observed between the DPSCs/VEGF and the DPSCs/Vector with regard to the proliferation characteristics of the cells; however, the DPSCs/VEGF showed a significantly enhanced expression of odontogenic differentiation genes and proteins. The results demonstrate that VEGF gene transfection may be a strategy to improve the efficacy of DPSCs for odontogenic differentiation. Further evaluation on the effectiveness of the VEGF gene in promoting the odontogenic differentiation of DPSCs in vivo is now required.

\section{Acknowledgements}

The authors would like to thank Dr Chenfei Zhang (Clinical Associate Professor in Endodontics, The University of Hong Kong) for his help in the initial phases of this study and the staff and postgraduate students of the Guanghua School of Stomatology, Sun Yat-sen University, who helped in the collection of clinical samples and the laboratory analysis. This study was supported by the Guangdong Medical Science Research Fund (B2012142) and the National Natural Science Foundation of China (81170932).

\section{References}

1. Gronthos S, Mankani M, Brahim J, Robey PG and Shi S: Postnatal human dental pulp stem cells (DPSCs) in vitro and in vivo. Proc Natl Acad Sci USA 97: 13625-13630, 2000.

2. Kawashima N: Characterisation of dental pulp stem cells: a new horizon for tissue regeneration? Arch Oral Biol 57: 1439-1458, 2012

3. Rodríguez-Lozano FJ, Bueno C, Insausti CL, et al: Mesenchymal stem cells derived from dental tissues. Int Endod J 44: 800-806, 2011.

4. Huang GT, Gronthos S and Shi S: Mesenchymal stem cells derived from dental tissues vs. those from other sources: their biology and role in regenerative medicine. J Dent Res 88: 792-806, 2009.

5. Yang X, van der Kraan PM, van den Dolder J, et al: STRO-1 selected rat dental pulp stem cells transfected with adenoviral-mediated human bone morphogenetic protein 2 gene show enhanced odontogenic differentiation. Tissue Eng 13: 2803-2812, 2007

6. Matsushita K, Motani R, Sakuta T, et al: The role of vascular endothelial growth factor in human dental pulp cells: induction of chemotaxis, proliferation, and differentiation and activation of the AP-1-dependent signaling pathway. J Dent Res 79: 1596-1603, 2000.

7. Tan YY, Yang YQ, Chai L, Wong RW and Rabie AB: Effects of vascular endothelial growth factor (VEGF) on MC3T3-E1. Orthod Craniofac Res 13: 223-228, 2010.

8. Lee JH, Um S, Jang JH and Seo BM: Effects of VEGF and FGF-2 on proliferation and differentiation of human periodontal ligament stem cells. Cell Tissue Res 348: 475-484, 2012.

9. D'Alimonte I, Nargi E, Mastrangelo F, et al: Vascular endothelial growth factor enhances in vitro proliferation and osteogenic differentiation of human dental pulp stem cells. J Biol Regul Homeost Agents 25: 57-69, 2011.

10. Wozney JM and Rosen V: Bone morphogenetic protein and bone morphogenetic protein gene family in bone formation and repair. Clin Orthop Relat Res: 26-37, 1998.

11. Samee M, Kasugai S, Kondo H, Ohya K, Shimokawa H and Kuroda S: Bone morphogenetic protein-2 (BMP-2) and vascular endothelial growth factor (VEGF) transfection to human periosteal cells enhances osteoblast differentiation and bone formation. J Pharmacol Sci 108: 18-31, 2008.

12. Liu B, Li X, Liang G and Liu X: VEGF expression in mesenchymal stem cells promotes bone formation of tissue-engineered bones. Mol Med Rep 4: 1121-1126, 2011.

13. Jiang J, Fan CY and Zeng BF: Osteogenic differentiation effects on rat bone marrow-derived mesenchymal stromal cells by lentivirus-mediated co-transfection of human BMP2 gene and VEGF165 gene. Biotechnol Lett 30: 197-203, 2008.

14. Bakopoulou A, Leyhausen G, Volk J, et al: Comparative analysis of in vitro osteo/odontogenic differentiation potential of human dental pulp stem cells (DPSCs) and stem cells from the apical papilla (SCAP). Arch Oral Biol 56: 709-721, 2011.

15. Lahmy R, Soleimani M, Sanati MH, Behmanesh M, Kouhkan F and Mobarra N: Pancreatic islet differentiation of human embryonic stem cells by microRNA overexpression. J Tissue Eng Regen Med. Jul 30, 2013 (Epud ahead of print). 
16. Hwang SY, Foley J, Numaga-Tomita T, Petranka JG, Bird GS and Putney JW, Jr: Deletion of Orail alters expression of multiple genes during osteoclast and osteoblast maturation. Cell Calcium 52: 488-500, 2012.

17. Pereira LO, Rubini MR, Silva JR, et al: Comparison of stem cell properties of cells isolated from normal and inflamed dental pulps. Int Endod J 45: 1080-1090, 2012.

18. Shi S and Gronthos S: Perivascular niche of postnatal mesenchymal stem cells in human bone marrow and dental pulp. J Bone Miner Res 18: 696-704, 2003.

19. Jiang L, Peng WW, Li LF, Yang Y and Zhu YQ: Isolation and identification of CXCR4-positive cells from human dental pulp cells. J Endod 38: 791-795, 2012.

20. Lee UL, Jeon SH, Park JY and Choung PH: Effect of platelet-rich plasma on dental stem cells derived from human impacted third molars. Regen Med 6: 67-79, 2011.

21. Trubiani O, Tripodi D, Delle Fratte T, Caputi S and Di Primio R: Human dental pulp vasculogenesis evaluated by CD34 antigen expression and morphological arrangement. J Dent Res 82: $742-747,2003$

22. Hata R and Senoo H: L-ascorbic acid 2-phosphate stimulates collagen accumulation, cell proliferation, and formation of a three-dimensional tissuelike substance by skin fibroblasts. J Cell Physiol 138: 8-16, 1989.

23. Hitomi K, Torii $\mathrm{Y}$ and Tsukagoshi N: Increase in the activity of alkaline phosphatase by L-ascorbic acid 2-phosphate in a human osteoblast cell line, HuO-3N1. J Nutr Sci Vitaminol (Tokyo) 38: 535-544, 1992
24. Torii Y, Hitomi K and Tsukagoshi N: L-ascorbic acid 2-phosphate promotes osteoblastic differentiation of MC3T3-E1 mediated by accumulation of type I collagen. J Nutr Sci Vitaminol (Tokyo) 40: 229-238, 1994.

25. Shima N, Kimoto M, Yamaguchi M and Yamagami S: Increased proliferation and replicative lifespan of isolated human corneal endothelial cells with L-ascorbic acid 2-phosphate. Invest Ophthalmol Vis Sci 52: 8711-8717, 2011.

26. MacDougall M, Gu TT and Simmons D: Dentin matrix protein-1, a candidate gene for dentinogenesis imperfecta. Connect Tissue Res 35: 267-272, 1996.

27. Ritchie HH, Berry JE, Somerman MJ, et al: Dentin sialoprotein (DSP) transcripts: developmentally-sustained expression in odontoblasts and transient expression in pre-ameloblasts. Eur J Oral Sci 105: 405-413, 1997.

28. Lee SY, Kim SY, Park SH, Kim JJ, Jang JH and Kim EC: Effects of recombinant dentin sialoprotein in dental pulp cells. J Dent Res 91: 407-412, 2012

29. Suzuki S, Sreenath T, Haruyama N, et al: Dentin sialoprotein and dentin phosphoprotein have distinct roles in dentin mineralization. Matrix Biol 28: 221-229, 2009. 